\title{
Relaxation of a colloidal particle into a nonequilibrium steady state
}

\author{
Valentin Blickle, ${ }^{1}$ Jakob Mehl, ${ }^{1}$ and Clemens Bechinger ${ }^{1,2}$ \\ 12. Physikalisches Institut, Universität Stuttgart, Pfaffenwaldring 57, 70550 Stuttgart, Germany \\ ${ }^{2}$ Max-Planck-Institut für Metallforschung, Heisenbergstrasse 3, 70569 Stuttgart, Germany
}

(Received 10 February 2009; revised manuscript received 24 April 2009; published 18 June 2009)

\begin{abstract}
We study the relaxation of a single colloidal sphere which is periodically driven between two nonequilibrium steady states. Experimentally, this is achieved by driving the particle along a toroidal trap imposed by scanned optical tweezers. We find that the relaxation time after which the probability distributions have been relaxed is identical to the decay of the velocity autocorrelation function measured in a steady state. In quantitative agreement with theoretical calculations the relaxation time strongly increases when driving the system further away from thermal equilibrium.
\end{abstract}

DOI: 10.1103/PhysRevE.79.060104

PACS number(s): 05.40.-a, 05.70.Ln, 82.70.Dd

The understanding of thermodynamic processes at small length scales is of central importance at the interface of physics, biology, and chemistry. Classical thermodynamics as originally developed for macroscopic systems with many internal degrees of freedom cannot be applied to, e.g., molecular machines, proteins, or micromechanical devices. This is because at microscopic scales, thermal fluctuations must not be neglected and the familiar well-defined thermodynamical quantities have to be replaced by corresponding distributions of finite width [1-3]. The situation is further complicated when these systems are driven out of thermal equilibrium as often encountered within their natural environment. The treatment of fluctuations in such nonequilibrium situations is even more difficult since it requires the full knowledge of the system's dynamics. Despite considerable progress in deriving exact relationships which are valid beyond thermal equilibrium [4-6] a comprehensive theoretical description of nonequilibrium is still lacking.

Among the huge manifold of nonequilibrium conditions, nonequilibrium steady states (NESS) are certainly the most simple conceivable situations, being characterized by a timeindependent probability distribution in the presence of a nonvanishing probability current. Accordingly, NESS present ideal conditions for fundamental studies and tests of nonequilibrium properties [7] on a microscopic scale [8,9].

In this Rapid Communication we experimentally investigate the relaxation behavior of a single colloidal particle which is periodically driven between two different nonequilibrium steady states NESS I and NESS II being created by scanning optical tweezers. We find that the NESS relaxation time as defined by the decay of the probability distribution only depends on the final state but is independent of the initial one. In addition, we show that this relaxation time is identical to that obtained by the decay of the velocity autocorrelation function in the steady-state regime, i.e., after relaxation has been completed. In agreement with theoretical calculations, the relaxation time increases when driving the system further away from thermal equilibrium.

The experimental setup has been already described elsewhere and will be discussed here only in brief [10]. Welldefined nonequilibrium steady states for a colloidal silica particle immersed in water with radius $a=0.65 \mu \mathrm{m}$ are created by scanning the highly focused beam of a $\mathrm{Nd}$ :YAG laser $(\lambda=532 \mathrm{~nm})$ along a circle with radius $R=1.14 \mu \mathrm{m}$ (see
Fig. 1). At rather high scanning frequencies the particle cannot follow the tweezers motion due to the viscous forces of the fluid, and it is confined to an effective three-dimensional toroidal optical trap. At intermediate scanning frequencies, however, each time the scanning laser focus passes the particle, a small displacement of the colloid along the scanning direction is induced. Since individual kicks are not resolvable by digital video microscopy [11], in this regime the scanning tweezers can be considered as exerting a constant force $f$ on the particle along the angular coordinate $x$ $[10,12,13]$. For a scanning frequency of $200 \mathrm{~Hz}$ and a laser intensity $I_{0} \approx 40 \mathrm{~mW}$ this leads to a drift velocity of $v \approx 7 \mu \mathrm{m} / \mathrm{s}$. In addition, the laser intensity is weakly modulated along the toroidal trap with an electro-optical device whose input signal is synchronized with the scanning motion of the laser focus. For a periodic intensity modulation $I(x)=I_{0}+\Delta I \sin (x)$ this leads to additional optical gradient forces, i.e., a static potential $V(x)=-\frac{V_{0}}{2} \sin (x+\phi)$ acting on the particle. The value of $\phi$ can be controlled by the relative phase difference between the scanned tweezers motion and its intensity variation. In total, the colloid is subjected to a tilted periodic potential $U(x)=V(x)-f R x$ corresponding to a NESS where $f$ and $V(x)$ can be tuned by $I_{0}$ and $\Delta I$, respectively. The driving force $f$ and $V(x)$ are not known a priori (a)

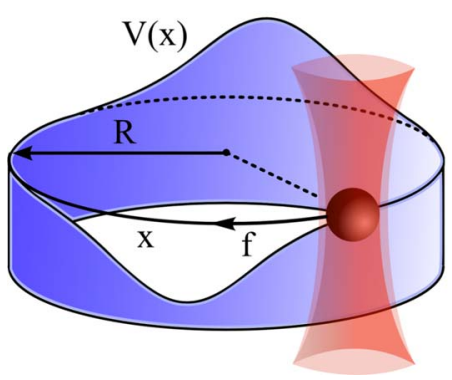

(b)

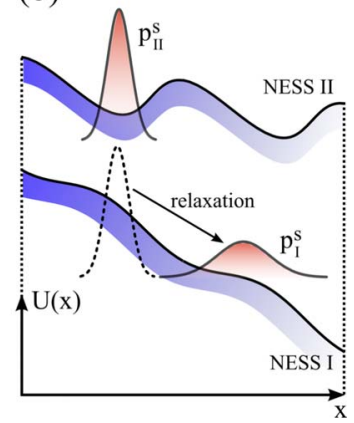

FIG. 1. (Color online) (a) Realization principle of the creation of a NESS for a colloidal particle by scanning a focused laser beam. (b) Schematic representation of NESS I and NESS II which correspond to a tilted periodic potential. A sudden change in the driving force $f$ and phase $\phi=\pi$ leads to a redistribution of the related probability distributions $p_{\mathrm{I}}^{s}$ and $p_{\mathrm{II}}^{s}$. 


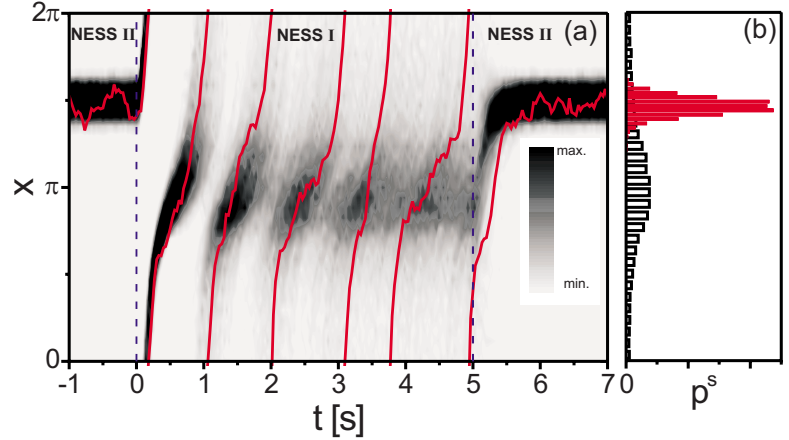

FIG. 2. (Color online) (a) Particle trajectory (solid line) and $p(x, t)$ as gray scaled background. Dashed vertical lines indicate the transitions between two different NESS with durations $t_{\mathrm{I}}=5 \mathrm{~s}$, $t_{\mathrm{II}}=2 \mathrm{~s}$ and a phase difference $\phi_{\mathrm{II}}=\pi$. (b) Normalized steady-state probability distributions of NESS I (open bars) and NESS II (closed bars).

but can be reconstructed via a generalized Boltzmann factor from the measured stationary probability distribution $p^{s}(x)$ and the probability current in the system [14].

The relaxation of a colloidal particle into a NESS is investigated by periodically toggling between two differing steady states. This is accomplished by a sudden change in the driving force $f$ and the phase $\phi$ according to the protocol

$$
\begin{gathered}
\text { if } 0 \leq t \leq t_{\mathrm{I}}: \quad f_{\mathrm{I}}, \phi_{\mathrm{I}}=0 \text { (NESS I), } \\
\text { if } t_{\mathrm{I}}<t \leq t_{\mathrm{I}}+t_{\mathrm{II}}: \quad f_{\mathrm{II}}, \phi_{\mathrm{II}} \text { (NESS II). }
\end{gathered}
$$

Unless otherwise stated, $V_{0}$ is kept constant at $V_{0} \approx 100 k_{\mathrm{B}} T$. The duration times $t_{\mathrm{I}}$ and $t_{\mathrm{II}}$ are chosen sufficiently long to allow the system to reach the corresponding stationary probability distributions $p_{\mathrm{I}}^{s}$ and $p_{\mathrm{II}}^{s}$. The entire protocol is typically repeated up to 800 times during each experiment to obtain adequate statistical averages.

To illustrate the principle of our experiments, we first discuss the situation where NESS II is close to thermal equilibrium. This is achieved by applying a rather weak driving force $f_{\mathrm{II}} \approx 4 k_{\mathrm{B}} T / \mu \mathrm{m}$. Accordingly, $U(x)$ exhibits a potential well of about $80 k_{\mathrm{B}} T$ where the particle remains strongly localized, thus closely resembling equilibrium conditions (locked state). This is clearly seen by the trajectory (solid line) in Fig. 2(a) which is confined to a small range of $x$ values. In contrast, NESS I has a much stronger force $f_{\mathrm{I}} \approx 53 k_{\mathrm{B}} T / \mu \mathrm{m}$. Therefore, $U(x)$ exhibits no local minimum and the particle is free to drift along the entire torus (running state).

Because of the superimposed Brownian motion, the particle trajectory varies between each cycle of the protocol. These fluctuations are taken into account by considering the probability distribution $p(x, t)$, i.e., the probability of finding the particle at time $t$ at position $x$. The measured $p(x, t) \mathrm{ob}-$ tained from about 800 cycles of the protocol is shown as gray scaled background of Fig. 2(a). For $-1<t<0 \mathrm{~s}$ the particle has relaxed to NESS II where it is localized inside the deep potential minimum. The corresponding strongly peaked steady-state probability distribution $p_{\mathrm{II}}^{s}$ is shown as closed bars in Fig. 2(b). Upon suddenly switching to NESS I

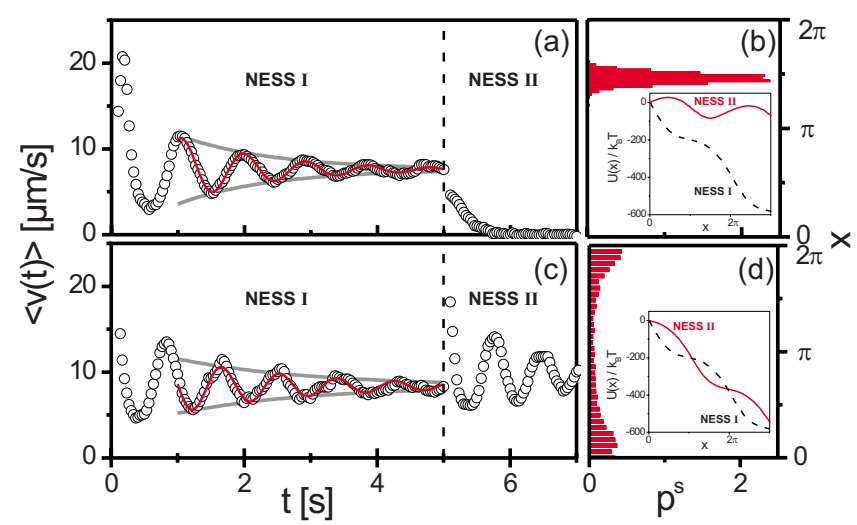

FIG. 3. (Color online) (a),(c) Open symbols: mean drift velocity $\langle v(t)\rangle$ after switching from NESS II to NESS I and vice versa $\left(f_{\mathrm{I}} \approx 53 k_{\mathrm{B}} T / \mu \mathrm{m}, \phi_{\mathrm{I}}=0\right)$. The NESS II parameters are given in Table I (a): (i), (c): (iv). Solid line: exponentially damped sinusoidal function. (b),(d): Corresponding potentials (inset) and steady-state distributions of NESS II.

at $t=0 \mathrm{~s}$, the particle starts to circulate along the entire toroidal trap; this leads to a broadening and a shift of the maximum in $p(x, t)$. The damped oscillatory behavior of $p(x=$ const,$t)$ is typical for the relaxation into a nonequilibrium steady state. This is in contrast to the situation at $t$ $=5 \mathrm{~s}$ when the protocol switches back to the equilibriumlike conditions of NESS II. Here, $p(x=$ const, $t)$ monotonically approaches its final value $p_{\mathrm{II}}^{s}$. It should be noted that the relaxation from NESS I into NESS II proceeds much more rapidly than into the other direction.

To quantify our findings we calculate the mean drift velocity $\langle v(t)\rangle=\left\langle\frac{x(t+\Delta t)-x(t-\Delta t)}{2 \Delta t}\right\rangle$, which is obtained by averaging the actual particle velocity $v(t)$ over several hundred cycles of the protocol. Since the length scale over which the potential $U(x)$ varies is more than 1 order of magnitude larger than the maximal particle displacement between two consecutive $(\Delta t=33 \mathrm{~ms})$ video frames, $\langle v(t)\rangle$ can be obtained from the experimentally determined trajectories. The symbols in Fig. 3(a) show $\langle v(t)\rangle$ for the same data set as in Fig. 2. After switching to NESS I, $\langle v(t)\rangle$ is a decaying oscillatory function which converges to the corresponding mean steady-state velocity. For $t>1 \mathrm{~s}$ it can be well described by an exponentially damped sinusoidal function (solid line) with decay time $\tau=1.4 \pm 0.2 \mathrm{~s}$ and the oscillation period given by the mean particle revolution time $T_{R}=0.9 \mathrm{~s}$. Similar as above, $\langle v(t)\rangle$ for the relaxation into the equilibriumlike NESS II is purely exponential with a decay time of $0.3 \mathrm{~s}$.

In order to understand how the relaxation into a NESS compares with that into thermal equilibrium we consider the relaxation time $\tau_{\mathrm{r}}^{\mathrm{eq}}$ of an overdamped Brownian particle into a parabolic potential. For this Uhlenbeck-Ornstein process the relaxation time is given by $[15,16]$

$$
\tau_{\mathrm{r}}^{\mathrm{eq}}=\frac{6 \pi \eta a}{k},
$$

where $\eta$ is the viscosity of the solvent, $k$ is the potential curvature, and $a$ is the particle radius. Obviously, $\tau_{\mathrm{r}}^{\mathrm{eq}}$ is entirely determined by the state into which the relaxation oc- 
TABLE I. Measured relaxation time of NESS I for different parameters of the initial NESS II.

\begin{tabular}{lcccc}
\hline \hline & $\begin{array}{c}f_{\mathrm{II}} \\
\left(k_{\mathrm{B}} T / \mu \mathrm{m}\right)\end{array}$ & $\phi_{\mathrm{II}}$ & $\begin{array}{c}\langle v\rangle_{\mathrm{II}} \\
(\mu \mathrm{m} / \mathrm{s})\end{array}$ & $\begin{array}{c}\tau \\
(\mathrm{s})\end{array}$ \\
\hline (i) & 4 & $\pi$ & 0 & $1.4 \pm 0.2$ \\
(ii) & 4 & 0 & 0 & $1.6 \pm 0.25$ \\
(iii) & 38 & $\pi$ & 3.4 & $1.6 \pm 0.25$ \\
(iv) & 52 & $\pi$ & 9.7 & $1.9 \pm 0.3$ \\
(v) & 99 & $\pi$ & 21 & $1.6 \pm 0.25$ \\
\hline \hline
\end{tabular}

curs and independent of the initial conditions. In order to investigate whether this holds also for relaxation processes into nonequilibrium states, we systematically vary the initial NESS II (by changing $f_{\mathrm{II}}$ and $\phi_{\mathrm{II}}$ ) and study the relaxation into the identical final NESS I (same parameters as in Fig. 2). As an example, Fig. 3(c) shows the relaxation for $f_{\mathrm{II}} \approx 52 k_{\mathrm{B}} T / \mu \mathrm{m}$ and $\phi_{\mathrm{II}}=\pi$. It should be realized that although the steady-state distribution of the initial state in Figs. 3(b) and 3(d) is rather different, the decay of the mean drift velocity is-within our experimental errors being caused by the finite number of trajectories and small optical driftsidentical. This is also seen in Table I, which summarizes five relaxation experiments from different NESS II into the identical NESS I. Within our experimental accuracy we observe the same relaxation time $\tau_{\mathrm{r}}=1.6 \pm 0.2 \mathrm{~s}$. At least in case of the specific NESS as considered here, this suggests that the relaxation time only depends on the final state. We confirmed the independence of the relaxation time for a variety of different NESS I conditions. Due to technical details all these experiments were performed in the running regime.

According to the fluctuation dissipation theorem, the temporal decay of fluctuations does not depend on whether they are imposed by an external force or spontaneously generated by the system itself. However, it is important to realize that this identity is only valid in or close to thermal equilibrium [17]. Therefore it is not a priori clear whether in a driven system (as considered here) $\tau_{\mathrm{r}}$, i.e., the decay time in response to a sudden change in the external driving force, is identical with the decay of the steady-state fluctuations. As shown in Fig. 4(a) the mean drift velocity autocorrelation function $C(t)=\left\langle v\left(t^{\prime}\right) v\left(t^{\prime}+t\right)\right\rangle_{t^{\prime}}$, obtained via a stationary measurement under NESS I conditions, is an exponentially decaying sinusoidal function with a decay time of $\tau_{\text {corr }}=1.7 \pm 0.2 \mathrm{~s}$. Within the experimental error this value is again identical with the above determined $\tau_{\mathrm{r}}$. To test whether this agreement is generally valid, we performed additional measurements with different driving forces $f$ and potential depths $V_{0}$. In Fig. 4(b) we compare $\tau_{\text {corr }}$ and $\tau_{\mathrm{r}}$ as measured for $40 k_{\mathrm{B}} T / \mu \mathrm{m}<f_{\mathrm{I}}<87 k_{\mathrm{B}} T / \mu \mathrm{m}$ and $V_{0}=100 k_{\mathrm{B}} T$ and $V_{0}=125 k_{\mathrm{B}} T$, respectively. The good agreement between the data points and the solid line (slope one) supports that $\tau_{\mathrm{r}}=\tau_{\text {corr }}$ and suggests that (as in equilibrium) the relaxation time of a NESS can be measured via transient or stationary measurements.

In order to compare the relaxation time with theory, we calculate $\tau_{\mathrm{r}}$ by numerically solving the Fokker-Planck equation [17]

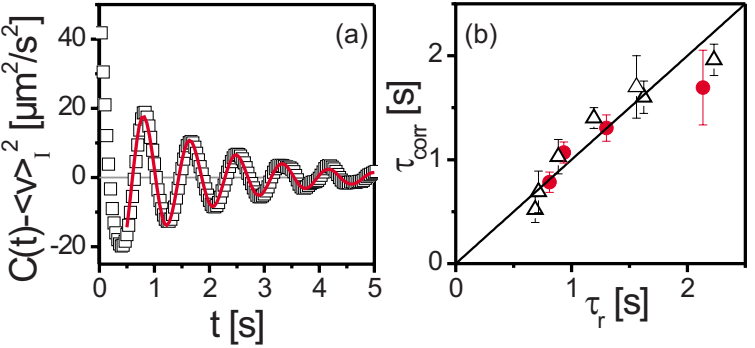

FIG. 4. (Color online) (a) Measured velocity autocorrelation function (symbols). The solid line is an exponentially decaying sinusoidal fit. (b) Relaxation time $\tau_{\mathrm{r}}$ vs $\tau_{\text {corr }}$ for different NESS where the driving force has been varied between 40 and $87 k_{\mathrm{B}} T / \mu \mathrm{m}$. The open and closed symbols correspond to potential depths of 125 and $100 k_{\mathrm{B}} T$, respectively. The straight line has slope one.

$$
\partial_{t} p(x, t)=-\partial_{x}\left[\mu_{0} F(x)-D_{0} \partial_{x}\right] p(x, t),
$$

with $R$ as the torus radius and $F(x)=-\frac{\partial U(x)}{\partial x}$ the total external force acting on the particle. The transport coefficients are assumed to be unaffected by the external driving force [3,9], therefore, the free diffusion coefficient $D_{0}$ and the mobility $\mu_{0}$ are taken from thermal equilibrium. In units of dimensionless time $\tilde{t}=\left(D_{0} / R^{2}\right) t \equiv \varepsilon^{-1} t$ and force $\widetilde{F}(x)=\left(R / k_{\mathrm{B}} T\right) F(x)$ the Fokker-Planck equation reduces to $\partial_{\tilde{t}} p(x, \widetilde{t})=\hat{L}_{x} p(x, \widetilde{t})$. Since the Fokker-Planck operator $\hat{L}_{x}=-\partial_{x} \tilde{F}(x)+\partial_{x}^{2}$ has no explicit time dependence, a separation ansatz for the probability distribution $p(x, \widetilde{t})=\sum_{n} \exp \left(-\lambda_{n} \widetilde{t}\right) q_{n}(x)$ leads to the following eigenvalue equation:

$$
-\lambda_{n} q_{n}(x)=\hat{L}_{x} q_{n}(x) .
$$

The relaxation of an arbitrary given initial probability distribution is described by the complete set of eigenvalues $\lambda_{n}$. However, in the long time limit only the two smallest eigenvalues $\lambda_{0}$ and $\lambda_{1}$ are relevant. The stationary solution $p^{s}(x)$ is given by $\lambda_{0}=0$ and $q_{0}(x)$. The real part of $\lambda_{1}, \mathcal{R}\left(\lambda_{1}\right) \equiv \tau_{1}^{-1}$ determines the asymptotic time dependence of the relaxation process. Therefore the relaxation time is $\varepsilon \tau_{1}$. Since Eq. (4) has no analytical solution, for the determination of the eigenvalues we have to expand the eigenfunctions into an orthonormal basis. Due to the periodic nature of the system a suitable choice is the Fourier series $q_{n}(x)=\frac{1}{\sqrt{2 \pi}} \sum_{l} c_{l}^{(n)} \exp (i l x)$. A straightforward calculation leads to

$$
-\lambda_{n} c_{k}^{(n)}=\sum_{l} L_{k l} c_{l}^{(n)}
$$

an eigenvalue equation for the matrix $\mathbf{L} \equiv\left(L_{k l}\right)$. In case of the experimentally realized sinusoidal potential, $\mathbf{L}$ is tridiagonal [18]. After truncating the size of the matrix to a finite value its eigenvalues are easily found using standard numerical algorithms.

Figure 5 shows the calculated $\varepsilon \tau_{1}$ (solid lines) as a function of the potential depth $V_{0}$ and for two different driving forces $f$. For large $V_{0}$ the system becomes equilibriumlike 


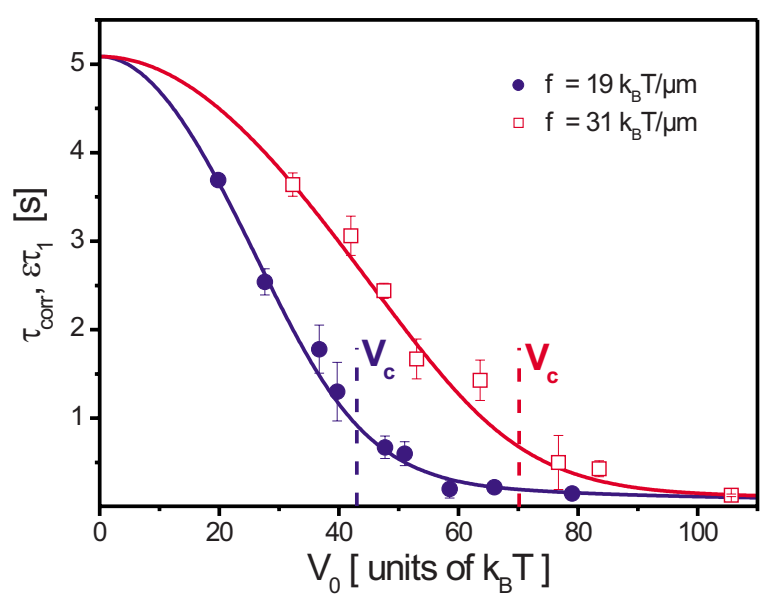

FIG. 5. (Color online) Symbols: measured decay times $\tau_{\text {corr }}$ for two different driving forces. The solid lines show the parameter-free numerical prediction for $\varepsilon \tau_{1}$. For potential depths smaller than the critical amplitude $V_{\mathrm{c}}=2 f R$ the minimum in the tilted potential vanishes, which leads to an enhancement of the diffusion coefficient [19].

and the relaxation time asymptotically approaches the Ornstein-Uhlenbeck result of Eq. (2). At small $V_{0}$ the relaxation time is only determined by the time scale the particle needs to diffuse along the toroidal trap, i.e., $\varepsilon \tau_{1}=D_{0} / R^{2}$.
These two limiting cases are connected via a monotonic curve. The closed symbols correspond to the experimentally determined decay time $\tau_{\text {corr }}$. The excellent parameter-free agreement between experimental and numerical results again supports our assumption that $\tau_{\text {corr }}$ is equal to $\tau_{\mathrm{r}}$ (see Fig. 4) and $a$ posteriori justifies that even for driven colloidal systems the Fokker-Planck equation, with $D_{0}$ taken from equilibrium, is still valid.

In summary, we have investigated the relaxation behavior of a colloidal particle into a NESS. Our results show that the NESS relaxation time is independent of the initial conditions from which the relaxation process starts. In agreement with calculations we confirm that, in case of a driven colloidal particle, the nonequilibrium relaxation time is identical to the decay time of the velocity autocorrelation function. It must be emphasized that it is not clear yet whether our observations are generally valid to arbitrary NESS or restricted to particular situations. We hope that our work will stimulate further theoretical studies in this direction. It will be also interesting to perform similar stationary nonequilibrium relaxation measurements in systems of sheared polymers [20] or vesicles [21].

We thank Udo Seifert and Thomas Speck for fruitful discussions and suggestions. V.B. was supported by the Deutsche Forschungsgemeinschaft (Grant No. BL-1067).
[1] C. Bustamante, J. Liphardt, and F. Ritort, Phys. Today 58 (7), 43 (2005).

[2] F. Ritort, Adv. Chem. Phys. 137, 31 (2008).

[3] U. Seifert, Eur. Phys. J. B 64, 423 (2008).

[4] C. Jarzynski, Phys. Rev. Lett. 78, 2690 (1997).

[5] J. Liphardt, S. Dumont, S. Smith, I. Tinoco, Jr., and C. Bustamante, Science 296, 1832 (2002).

[6] U. Seifert, Phys. Rev. Lett. 95, 040602 (2005).

[7] Y. Oono and M. Paniconi, Prog. Theor. Phys. Suppl. 130, 29 (1998).

[8] T. Hatano and S. I. Sasa, Phys. Rev. Lett. 86, 3463 (2001).

[9] T. Speck and U. Seifert, Europhys. Lett. 74, 391 (2006).

[10] V. Blickle, T. Speck, C. Lutz, U. Seifert, and C. Bechinger, Phys. Rev. Lett. 98, 210601 (2007).

[11] The particle trajectory is monitored with a spatial and temporal resolution of $20 \mathrm{~nm}$ and $33 \mathrm{~ms}$, respectively.

[12] L. P. Faucheux, G. Stolovitzky, and A. Libchaber, Phys. Rev. E 51, 5239 (1995).
[13] C. Lutz, M. Reichert, H. Stark, and C. Bechinger, Europhys. Lett. 74, 719 (2006).

[14] V. Blickle, T. Speck, U. Seifert, and C. Bechinger, Phys. Rev. E 75, 060101(R) (2007).

[15] G. Uhlenbeck and L. Ornstein, Phys. Rev. 36, 823 (1930).

[16] C. Gardiner, Handbook of Stochastic Methods (Springer, Berlin, 2004).

[17] H. Risken, The Fokker-Planck Equation (Springer, Berlin, 1996).

[18] J. Mehl, T. Speck, and U. Seifert, Phys. Rev. E 78, 011123 (2008).

[19] P. Reimann, C. Van den Broeck, H. Linke, P. Hanggi, J. M. Rubi, and A. Perez-Madrid, Phys. Rev. Lett. 87, 010602 (2001).

[20] S. Gerashchenko and V. Steinberg, Phys. Rev. E 78, 040801(R) (2008).

[21] V. Kantsler, E. Segre, and V. Steinberg, Phys. Rev. Lett. 101, 048101 (2008). 